\title{
Long-term microstructure and cerebral blood flow changes in patients recovered from COVID-19 without neurological manifestations
}

\author{
Yuanyuan Qin, ${ }^{1}$ Jinfeng Wu, ${ }^{2}$ Tao Chen, ${ }^{3}$ Jia Li, ${ }^{1}$ Guiling Zhang, ${ }^{1}$ Di Wu, ${ }^{1}$ Yiran Zhou, ${ }^{1}$ Ning Zheng, ${ }^{2}$ Aoling Cai, ${ }^{2}$ Qin Ning, ${ }^{3}$ \\ Anne Manyande, ${ }^{4}$ Fuqiang $\mathrm{Xu}^{2,5}{ }^{2}$ Jie Wang, ${ }^{2,5}$ and Wenzhen Zhu' \\ 'Department of Radiology, Tongji Hospital, Tongji Medical College, Huazhong University of Science and Technology, Wuhan, Hubei, China. 2State Key Laboratory of Magnetic Resonance and Atomic and \\ Molecular Physics, Key Laboratory of Magnetic Resonance in Biological Systems, Innovation Academy for Precision Measurement Science and Technology, Chinese Academy of Sciences, Wuhan, Hubei, \\ China. ${ }^{3}$ nstitute and Department of Infectious Disease, Tongji Hospital, Tongji Medical College, Huazhong University of Science and Technology, Wuhan, Hubei, China. ${ }^{4}$ School of Human and Social Sciences, \\ University of West London, Middlesex, United Kingdom. ${ }^{5}$ University of Chinese Academy of Sciences, Beijing, China.
}

BACKGROUND. The coronavirus disease 2019 (COVID-19) rapidly progressed to a global pandemic. Although some patients totally recover from COVID-19 pneumonia, the disease's long-term effects on the brain still need to be explored.

METHODS. We recruited 51 patients with 2 subtypes of COVID-19 (19 mild and 32 severe) with no specific neurological manifestations at the acute stage and no obvious lesions on the conventional MRI 3 months after discharge. Changes in gray matter morphometry, cerebral blood flow (CBF), and white matter (WM) microstructure were investigated using MRI. The relationship between brain imaging measurements and inflammation markers was further analyzed.

\begin{abstract}
RESULTS. Compared with healthy controls, the decrease in cortical thickness/CBF and the changes in WM microstructure were more severe in patients with severe disease than in those with mild disease, especially in the frontal and limbic systems. Furthermore, changes in brain microstructure, $C B F$, and tract parameters were significantly correlated $(P<0.05)$ with the inflammatory markers C-reactive protein, procalcitonin, and interleukin 6.

CONCLUSION. Indirect injury related to inflammatory storm may damage the brain, altering cerebral volume, CBF, and WM tracts. COVID-19-related hypoxemia and dysfunction of vascular endothelium may also contribute to neurological changes. The abnormalities in these brain areas need to be monitored during recovery, which could help clinicians understand the potential neurological sequelae of COVID-19.
\end{abstract}

FUNDING. Natural Science Foundation of China.

\section{Introduction}

The highly infectious coronavirus disease 2019 (COVID-19), caused by severe acute respiratory syndrome coronavirus 2 (SARS-CoV-2) rapidly progressed to a global pandemic and unleashed widespread concern around the world in 2020. To date, COVID-19 has infected more than 79 million people and killed over 1.7 million worldwide (1). The prominent symptoms of COVID-19 are in the respiratory system. However, growing evidence indicates that SARS-CoV-2 not only attacks the lungs, kidneys, and heart, but also affects the central nervous system (CNS)

\section{Related Commentary: https://doi.org/10.1172/JCl148376}

Authorship note: YQ, J Wu, and TC contributed equally to this work. YQ, J Wu, and TC are co-first authors.

Conflict of interest: The authors have declared that no conflict of interest exists. Copyright: () 2021, American Society for Clinical Investigation.

Submitted: January 7, 2021; Accepted: February 23, 2021; Published: April 15, 2021

Reference information: J Clin Invest. 2021;131(8):e147329.

https://doi.org/10.1172/JCl147329. at the early stage of infection (2, 3). COVID-19-associated neurological manifestations such as encephalitis, acute necrotizing encephalopathy (ANE), demyelination, cerebrovascular disease, and Guillain-Barre syndrome and its variants have been reported $(2,4-10)$. The underlying mechanisms are potentially related to direct virus infection of the CNS, virus-induced hyperinflammatory and hypercoagulable states, and postinfectious immunemediated processes $(3,11)$. However, previous studies are based on retrospective hospitalized data and focus on the immediate effects of COVID-19, leaving the long-term impact on the brain still unknown (12). The clinical spectrum of COVID-19 ranges from mild to severe to critically ill, according to WHO guidelines (13). Critically ill patients, who experience a more severe hypoxia state and inflammatory storm, often have more severe nervous system injury and systemic complications, whereas patients with mild and severe disease types have no specific neurological symptoms at the acute stage and experience relatively better clinical outcomes $(13,14)$. Although some patients totally recovered from COVID-19 pneumonia, whether there was potential damage to the brain or not remains unknown. Thus, the long-term impact on 


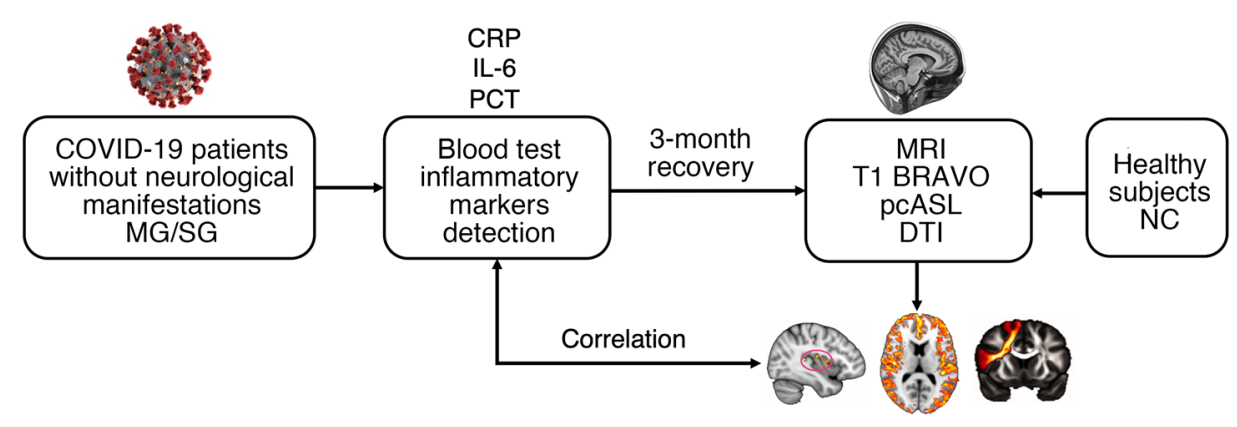

Figure 1. Flow diagram of the experimental design. MG, mild group; SG, severe group; CRP, C-reactive protein; IL-6, interleukin-6; PCT, procalcitonin; MRI, magnetic resonance imaging; BRAVO, brain volume; pcASL, pseudo-continuous arterial spin labeling; DTI, diffusion tensor imaging; NC, normal control.

the brain of patients without any specific neurological manifestations of COVID-19 needs to be further investigated, which could yield better understanding of the ongoing neurological and psychological effects of the disease and generate clinical guidance toward complete recovery.

In the current study, we aimed to explore the neurological alterations after long-term recovery in patients who had COVID-19 with no specific neurological manifestations at the acute stage and at 3-month follow-up. Patients diagnosed as having mild and severe types of COVID-19 infection according to WHO guidelines and who had no specific neurological manifestations during hospitalization were enrolled and followed for 3 months. The potential changes in brain microstructure and cerebral blood flow (CBF) were investigated and compared with age- and sex-matched healthy controls using quantitative MRI and state-of-the-art postprocessing protocols.

\section{Results}

All patients were enrolled according to the study design shown in Figure 1. Briefly, the participating patients were divided into the mild group (MG) and severe group (SG) according to WHO guidelines. There were no specific neurological manifestations at the acute stage. Inflammatory markers and other demographics and behavioral measures were applied during hospitalization when patients were sick. At 3 months after recovery, multiple model MRI methods were used to investigate changes in the brain structure and blood flow.

Demographics and behavioral measures. As shown in Table 1, 51 patients recovered from COVID-19 were recruited for the study; 19 patients were assigned to the MG and 32 patients were assigned to the SG. Results of the mean hematocrit (Hct) value showed no significant difference between the MG and SG ( $P=$ 0.835). For males only, the Hct value showed no significant difference between the MG and SG $(P=0.923)$. Results were similar for females $(P=0.553)$. This could reduce the Hct effect on $\mathrm{CBF}$ and reflect more on the impact of the disease in each group. For inflammatory markers, we found significant elevation of C-reactive protein $(\mathrm{CRP})(P<0.001)$, procalcitonin $(\mathrm{PCT})(P=$ $0.001)$, and interleukin-6 (IL-6) $(P=0.041)$ in the SG compared with the MG. All patients recovered from COVID-19 pneumonia were diagnosed and hospitalized in March 2020. According to their medical histories, patients showed no specific neurologi- cal symptoms during hospitalization. They experienced coughing, fever, dyspnea, diarrhea, headache, fatigue, myalgia, and chest tightness during the period of SARS-CoV-2 infection. All patients exhibited lesion absorption of the lungs. However, compared with the MG, the SG had more sequelae in the lungs, such as fiber strands and bronchiectasis. Supplemental Figure 1, A-D illustrates 2 patients with mild and severe types of COVID-19 at baseline and 3 months after discharge. To avoid cross-infection, the brain MRI scans were not performed during hospitalization in March, but instead in late June and early July, which is about 3 months after discharge. All patients showed no obvious lesions on the conventional MRI. The mean duration from discharge to MRI scanning was 101.21 \pm 12.24 days. Another 31 age-, sex-, and education-matched non-COVID-19 volunteers were enrolled to the normal control (NC) group.

Cortical thickness and subcortical volume comparisons. Compared with the NC group, the SG showed significantly (voxel-wise permutation test, $P<0.05)$ reduced cortical thickness in the left insula (Figure 2A) (cluster volume [CV]: $1742 \mathrm{~mm}^{3}$, peak Montreal Neurological Institute [MNI] coordinates: $-52-14$ 12, peak $t$ value $[t]$ : 5.4172), left hippocampus (CV: $327 \mathrm{~mm}^{3}$, MNI: $-25-11$ $-12, t: 4.178$ ), and left superior temporal gyrus (CV: $366 \mathrm{~mm}^{3}$, MNI: $-40-3511, t: 5.2035)$. No significant differences were observed between the NC group and the MG, or between the MG and SG. Correlational analysis further revealed that the cortical thickness of the left hippocampus was negatively correlated with the PCT in the SG (Figure 2B).

In subcortical nuclei comparisons, 14 nuclei volumes were obtained from preprocessing. Namely, the left caudate, right caudate, left putamen, right putamen, left thalamus, right thalamus, left globus pallidus, right globus pallidus, left hippocampus, right hippocampus, left amygdala, right amygdala, left accumbens, and right accumbens. For group comparisons, the SG tended to have significantly reduced volumes of the left putamen $(P=0.0202)$, left thalamus $(P=0.0370)$, and right thalamus ( $P=0.0084$ ) compared with the NC group (Figure 2C). The SG was also found to show significantly decreased volumes of the left caudate $(P=0.0368)$ and left putamen $(P=0.0284)$ compared with the MG (Figure 2D).

$C B F$ comparison. For the SG, CBF values were generally and significantly $(P<0.05$, voxel-wise permutation test) lower compared with the NC group across the gray matter cortex and the 
Table 1. Demographic information of recovered COVID-19 groups and the control group

\begin{tabular}{|c|c|c|c|c|c|c|}
\hline & \multicolumn{2}{|c|}{ COVID-19 group ( $n=51)$} & \multirow{2}{*}{$\begin{array}{l}\text { Control group } \\
\qquad(n=31)\end{array}$} & \multirow[t]{2}{*}{$P$, (MG-NC) } & \multirow[t]{2}{*}{$P$, (SG-NC) } & \multirow[t]{2}{*}{$P,(\mathrm{MG}-\mathrm{SC})$} \\
\hline & Mild $(n=19)$ & Severe $(n=32)$ & & & & \\
\hline Age, years & $59.37 \pm 5.87$ & $63.19 \pm 5.37$ & $60.58 \pm 6.42$ & $0.507^{A}$ & $0.085^{A}$ & $0.022^{A}$ \\
\hline Sex, male/female & $7 / 12$ & $16 / 16$ & $18 / 13$ & $0.774^{\mathrm{B}}$ & $0.616^{B}$ & $0.398^{\mathrm{B}}$ \\
\hline Handedness, right/left & $19 / 0$ & $32 / 0$ & $31 / 0$ & - & - & - \\
\hline Hematocrit $(\%)$ & $44.69 \pm 3.05$ & $44.92 \pm 3.95$ & - & - & - & $0.835^{A}$ \\
\hline Diabetes & $2(11)$ & $7(22)$ & $2(6)$ & $0.606^{B}$ & $0.080^{B}$ & $0.304^{\mathrm{B}}$ \\
\hline Coronary heart disease & $1(5)$ & $3(9)$ & $1(3)$ & $0.722^{\mathrm{B}}$ & $0.668^{\mathrm{B}}$ & $0.597^{B}$ \\
\hline Symptoms, $n(\%)$ & & & - & & & \\
\hline Cough & $13(68)$ & $25(78)$ & - & & & \\
\hline Fever & $16(84)$ & $27(84)$ & - & & & \\
\hline Myalgia & $2(11)$ & $6(19)$ & - & & & \\
\hline Chest tightness & $5(26)$ & $14(43)$ & - & & & \\
\hline Inflammatory markers & & & - & & & \\
\hline CRP & $12.07 \pm 20.03$ & $91.40 \pm 63.14$ & - & - & - & $0.000^{A}$ \\
\hline PCT & $0.04 \pm 0.02$ & $0.14 \pm 0.12$ & - & - & - & $0.001^{A}$ \\
\hline IL-6 & $5.63 \pm 8.87$ & $28.68 \pm 43.06$ & - & - & - & $0.041^{A}$ \\
\hline
\end{tabular}

The significant differences are labeled in bold. $P<0.05$. Data are mean \pm SD. ${ }^{A}$ Significance of 2 -sample $t$ test. ${ }^{B}$ Significance of $\chi^{2}$ test.

peak value was observed in the left insula (Figure 3A, MNI: -43 -6 1, $t:$ 6.0816). Further, the peak region insula was manually extracted to conduct the correlational analysis with the level of inflammatory markers. Compared with the MG (Figure 3B), the SG exhibited significantly lower $\mathrm{CBF}$ values in the bilateral superior medial frontal gyrus (CV: $3417 \mathrm{~mm}^{3}$, MNI: 151 24, $t$ : 5.6017), left insula (CV: $2650 \mathrm{~mm}^{3}$, MNI: $\left.-43-11-9, t: 5.3929\right)$ and right insula (CV: $381 \mathrm{~mm}^{3}$, MNI: $\left.43-152, t: 5.377\right)$. However, there were no significant differences between the NC group and the MG. Results showed that the mean CBF of the left insula in the SG was positively correlated with the level of PCT (Figure 3C).

For subcortical nuclei $\mathrm{CBF}$ alterations, compared with the NC group, the SG exhibited significantly reduced CBF values in the left caudate $(P=0.0460)$, right caudate $(P=0.0140)$, left putamen $(P=0.0344)$, right putamen $(P=0.0048)$, right globus pallidus $(P=0.0106)$, right hippocampus $(P=0.0166)$, right amygdala $(P=0.0160)$, and right accumbens $(P=0.0194$; Figure 3D). Compared with the $\mathrm{MG}$, the SG showed lower CBF values in the left caudate $(P=0.0346)$, right caudate $(P=0.0162)$, right globus pallidus $(P=0.0208)$, and right amygdala $(P=0.0258)$ (Figure 3E). There were no significant differences observed between the NC group and the MG.

White matter XTRACT analysis. For a specific tract estimated from a given subject, the shape and position of that tract differed slightly, and it was inappropriate to conduct the spatially corresponding voxel-wise comparison. For a given tract, XTRACT yielded multiple postanalysis statistics. Three meaningful statistics are exhibited, i.e., volume, length, and fractional anisotropy
(FA). Specifically, the volume is a voxel-wise statistic. Every voxel that met the threshold of the estimation of probabilistic diffusion tractography was taken into account and the total voxel-wise trajectory size was considered the volume. The volume suggests the most accurate and sensitive statistic in a given tract, and it was taken as the optimal quantitative statistic. The statistic length follows the same logic, but due to the slightly different shape of a given trajectory, the length was not considered as the optimal statistic. FA is a rough tract-wise indicator, revealing only vague and suggestive information about that specific tract. The significant comparisons between groups are marked and exhibited in Supplemental Table 1; supplemental material available online with this article; https://doi.org/10.1172/JCI147329DS1. We found widespread decreases in volume, length, and mean FA values in association, commissural, projection, and limbic fiber bundles in the MG and SG when compared with the NC group.

Compared with the NC group, the MG showed 17 tract changes in 3 different measures (volume: 7; length: 8; FA: 2; Supplemental Table 1). For parameter volume, the MG had overall lower values in the left acoustic radiation $(\mathrm{AR})(P=0.0076)$, right anterior thalamic radiation (ATR) $(P=0.0264)$, left cingulum bundle, dorsal (CBD) $(P=0.0410)$, right frontal aslant tract (FAT) $(P=0.0464)$, forceps minor $(\mathrm{FMI})(P=0.0410)$, left inferior longitudinal fasciculus (ILF) $(P=0.0084)$, and right ILF $(P=0.0094$; Figure $4 \mathrm{~A})$. Using these 17 different tract measures, the first principal component (PC1) was obtained, which represents $35.1 \%$ of the total variance of the different parameters. The correlation analysis indicated that PC1 was only significantly correlated with 1 of the 3 
A

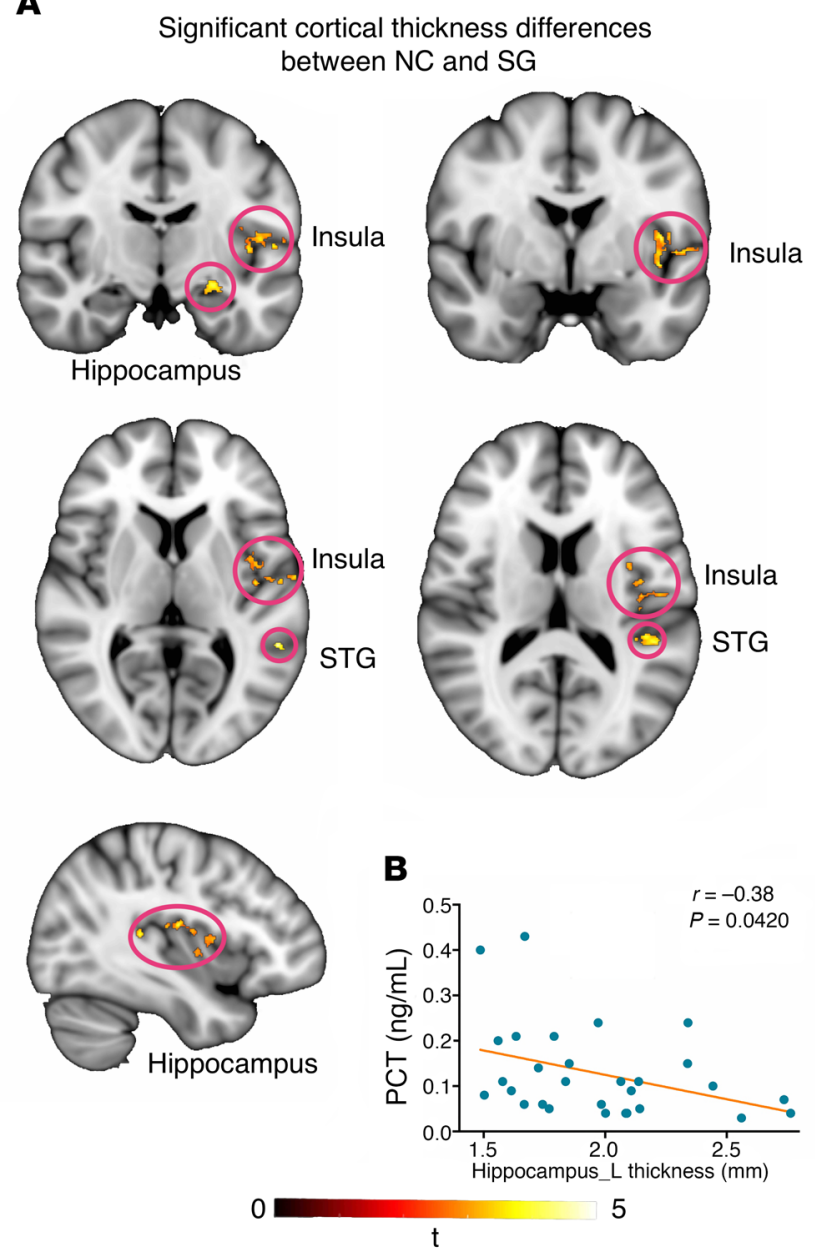

C
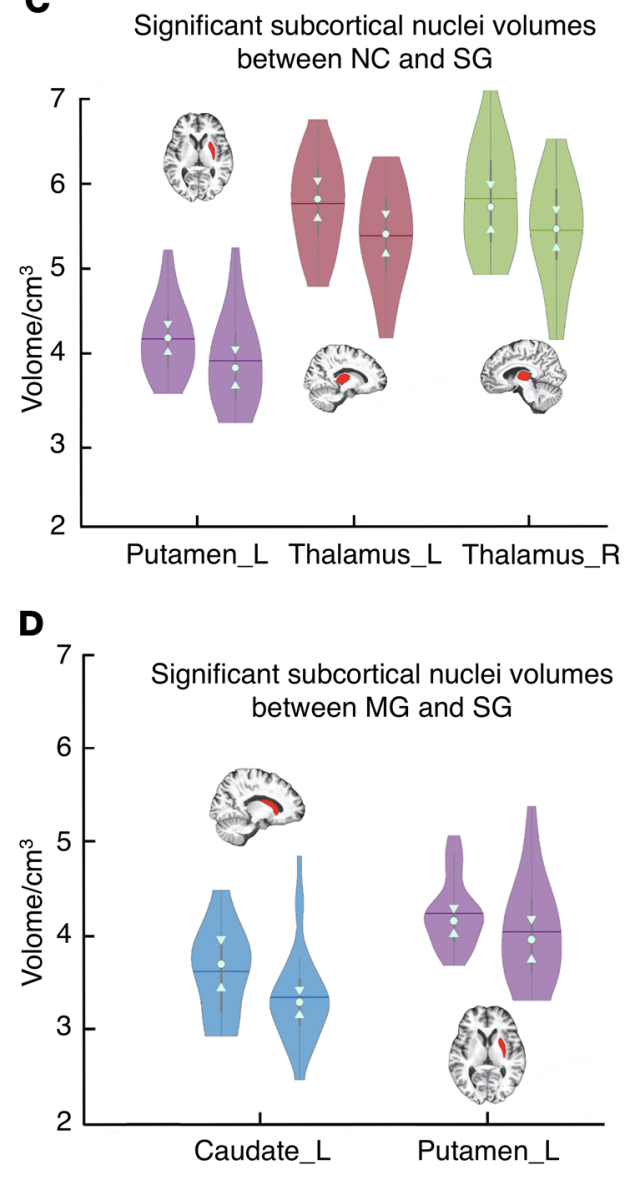

Figure 2. Cortical and subcortical morphology analyses of recovered COVID-19 patients. (A) Significant cortical thickness differences between the NC group and SG. (B) Significant negative correlations between the left hippocampus thickness and inflammatory marker PCT values in the SG ( $r=-0.38$, $P=0.0420$ ). Results of subcortical nuclei volume comparisons between the NC group and the SG (C) (left: NC; right: SG), and the MC and SG (D) (left: MG; right: SC), as exhibited in the violin plot. The trunk size shows the probability density of the data at different values; the bigger the trunk, the denser the values appeared at that level. The notch indicates the $25 \%$ median and $75 \%$ interquartile range. The mean value of the group is shown by solid line. The bigger the standard deviation, the thinner the violin plot. The brain slices of subcortical nuclei were extracted from a subject. Left violin plots indicate the NC/MG; right violin plots indicate the SG. STG, superior temporal gyrus. Sample sizes: NC, $n=31 ; \mathrm{MG}, n=19 ; \mathrm{SG}, n=32$. Permutation test, $P<0.05$.

inflammatory markers, namely PCT $(P=0.0483$, Figure $4 \mathrm{~B})$. To calculate the contribution of the different tracts, the correlational analysis between the different parameters and PCT was calculated, which revealed that the volumes of the right ATR (Figure 4C) and right ILF (Figure 4D) were negatively and significantly correlated with the PCT in the MG.

Compared with the NC group, the SG showed 33 tract changes in 3 different parameters (volume: 12; length: 5; FA: 16; Supplemental Table 1). For parameter volume, the SG showed overall lower values in the right ATR $(P=0.0134)$, left CBD $(P=$ 0.0076), right CBD $(P=0.0312)$, left corticospinal tract (CST) $(P=0.0412)$, right FAT $(P=0.0284)$, FMI $(P=0.0130)$, left fornix (FX) $(P=0.0232)$, right inferior fronto-occipital fasciculus (IFO) $(P=0.0162)$, left ILF $(P=0.0046)$, right ILF $(P=0.0014)$, right optic radiation $(\mathrm{OR})(P=0.0146)$, and left superior longitudinal fasciculus II (SLF2) $(P=0.0206$; Figure 5$)$. Note that the volumes of the right ATR, left CBD, right FAT, FMI, left ILF, and right ILF were decreased in the MG and the SG compared with the NC group. With these changed tracts $(n=33)$, PC1 was calculated, which represents $26.1 \%$ of the total variance of the parameters. The correlation analysis showed that $\mathrm{PC} 1$ was only significantly correlated with IL-6 $(P=0.0430$; Figure $6 \mathrm{~A})$. To calculate the contribution of the different white matters, the correlations of different tracts and IL- 6 were calculated. Correlational analysis revealed that the volumes of the left CST and the right OR were significantly positively correlated with IL-6. Moreover, the FA on the left of the middle longitudinal fasciculus (MDLF) were also significantly positively correlated with IL-6 (Figure 6, B-D).

\section{Discussion}

Our findings demonstrated that the patients with mild- and severe-type COVID-19 with no specific neurological manifestations or obvious lesions on the conventional MRI, although totally recovered from pneumonia, still exhibited brain microstructure changes and decrease in CBF after a 3-month follow-up. Com- 
A

Significant cortical CBF differences between NC and SG
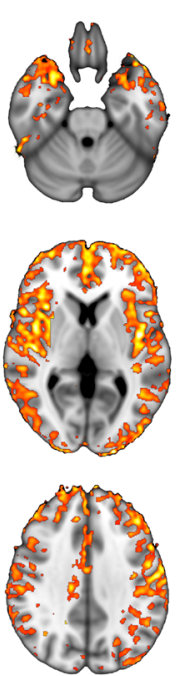
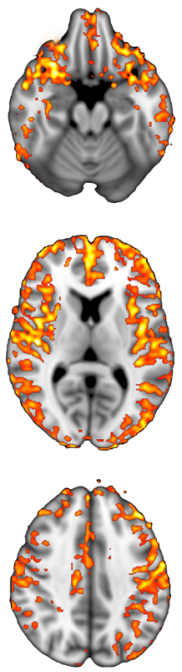
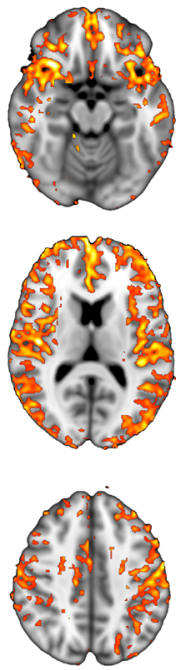
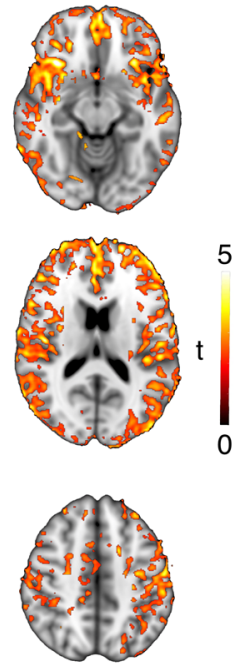

B

Significant cortical CBF differences between $M G$ and $S G$
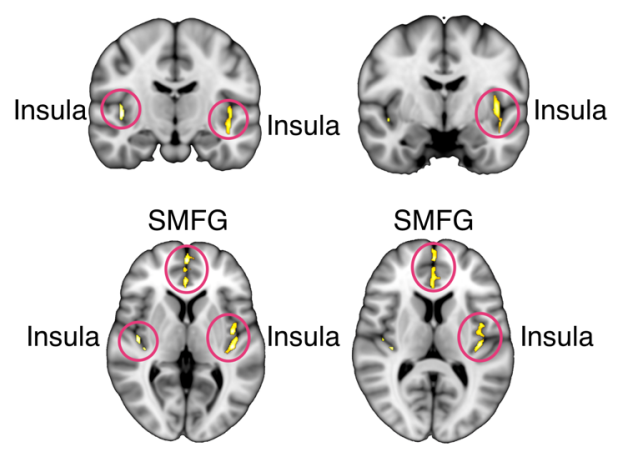

C

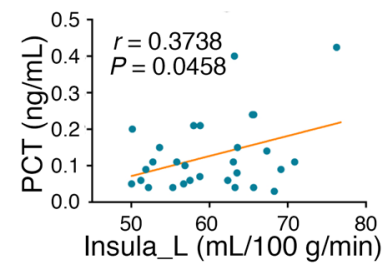

D

Significant subcortical nuclei CBF differences between NC and SG

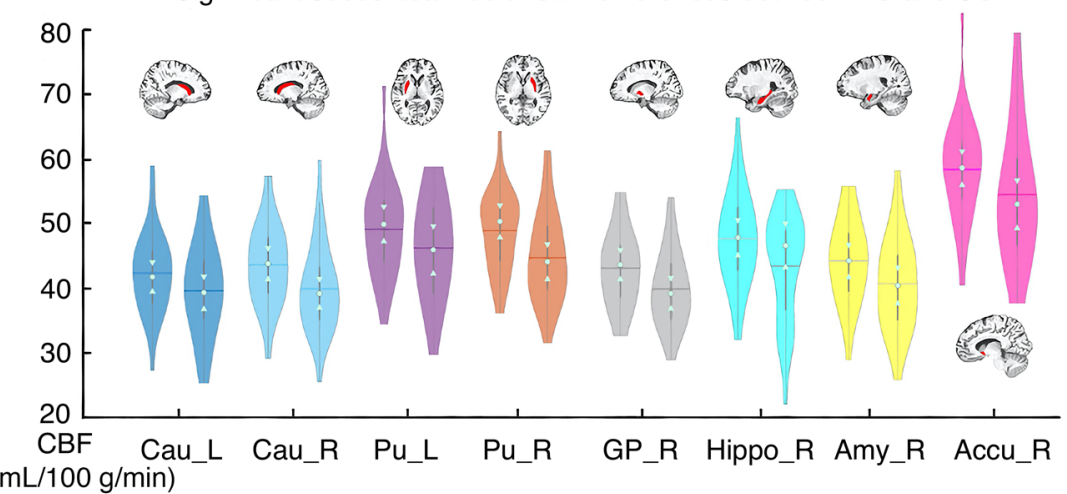

E

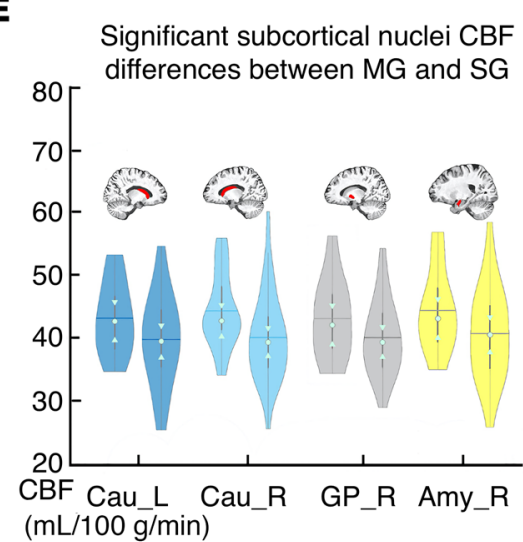

Figure 3. Significant differences in cortical and subcortical CBF between groups. (A) NC group ( $n=31)$ and SG $(n=32)$. (B) MG ( $n=19)$ and SG ( $n=32)$. (C) Significant positive correlation between the left insula CBF and inflammatory marker PCT values in the SC $(r=0.3738, P=0.0458)$. Results of the comparisons of the CBF in the subcortical nuclei between groups: (D) NC group and SG; (E) MG and SG. SMFG, superior medial frontal gyrus. The left of the brain images is actually the right side of the brain. Accu, accumbens; Amy, amygdala; Cau, caudate; GP, globus pallidus; Hippo, hippocampus; Pu, putamen; L, left; R, right. Left violin plots indicate NC/MG, right violin plots indicate SG. Permutation test, $P<0.05$.

pared with healthy controls, just a few changes were found in the white matter tract in the MG, with no significant changes in gray matter and CBF. The decrease in cortical thickness, changes in white matter microstructure, and decrease in regional $\mathrm{CBF}$ were much more profound and extensive in the SG than in the MG, especially in the frontal and limbic systems. Furthermore, the brain microstructure changes and CBF decrease were highly correlated with the level of inflammatory markers in the SG. The abnormalities in these brain areas could help clinicians to understand the potential neurological sequelae of COVID-19.

Cortical abnormality. Brain integrity appears to be vulnerable to systemic inflammation during critical illness. In our study, the SG had significantly lower cortical thickness in the left insula, left hippocampus, and left superior temporal gyrus compared with the NC group, but no significant differences were seen between the MG and the NC group. A study by Lindlau et al. (15) suggested that high levels of the PCT and IL-6 in the blood serum of critically ill patients are associated with a higher likelihood of hippocampal atrophy 6 months after discharge from the intensive care unit. Similarly, we also found that atrophy of the hippocampus was negatively correlated with PCT in recovered COVID-19 patients. The hippocampus plays a key role in both memory formation and spatial navigation and has the capacity to support flexible cognition and behavior (16). The hippocampal volume has also been shown to be sensitive to stress severity (17). In our study, COVID-19 infection was a very stressful event for patients, and even for their family members. Thus, hippocampal impairment could be attributed partly to the influence of a stressful event and partly to hypoxia or other processes. A recent retrospective study on brain MRI findings in acute SARS- 
$\mathbf{A}$

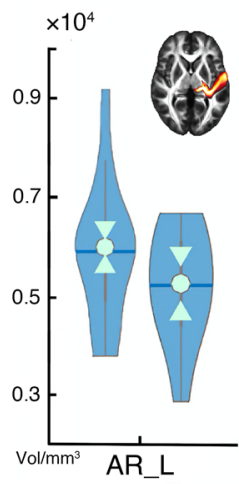

Significant tracts volumes between NC and MG
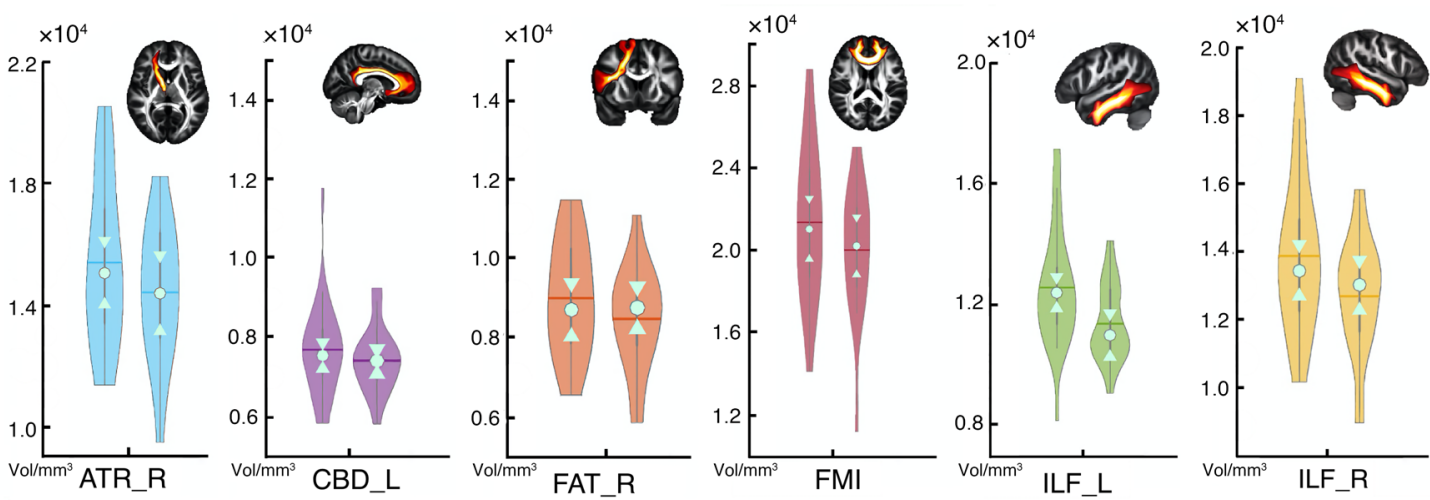

Correlations between tracts parameters and inflammatory markers in MG

B

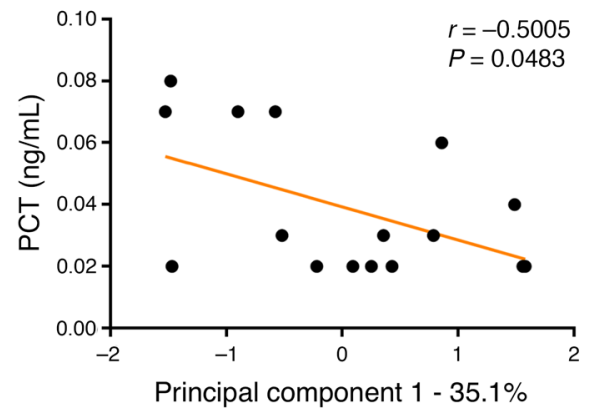

C

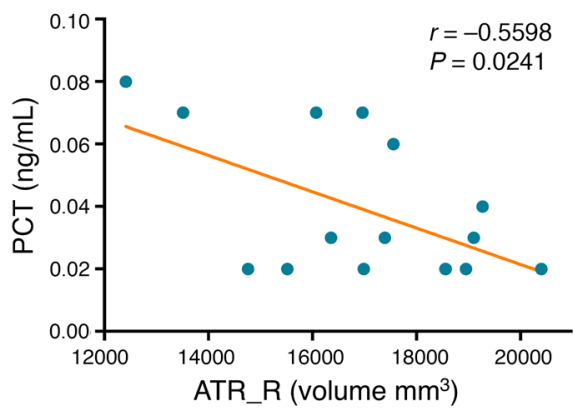

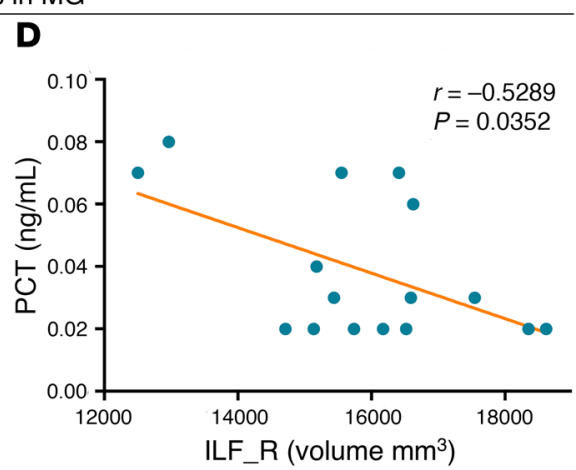

Figure 4. Group comparisons and correlation analyses in the MG. (A) Significant difference in tract volume between the NC group ( $n=31)$ and the MC $(n=19)$. Permutation test, $P<0.05$. The brain slice of a specific tract from HCP population is displayed in the right corner. For standardization, these tracts were extracted from the HCP population instead of the subjects' specific tracts. The PC1 (B) and the volumes of the right ATR (C) and right ILF (D) showed a significant correlation with the PCT level in the MC $(P<0.05)$. Left violin plots indicate the NC group; right violin plots indicate the MG.

CoV-2 infection indicated that signal abnormalities in the medial temporal lobe appeared more frequently in patients with severe COVID-19 (18), such as in the case of herpesvirus encephalitis or autoimmune limbic encephalitis. With no acute neurological manifestations, the recovered severe-type patients still showed gray matter atrophy in the left insula, left hippocampus, and left superior temporal gyrus in this study, which are all important components of the limbic system. Our study indicates a possible vulnerability of the limbic system for COVID-19 infection, which may be partly aligned with the angiotensin converting enzyme 2-rich (ACE-2-rich) region in the brain.

In the current study, the CBF in the SG was generally lower across the bilateral frontal and temporal cortex than in the NC group, with the lowest region in the left insula. In line with a previous study (19), the SG with bilateral frontal-temporal involvement may suffer much more severe hypoxic state, which may be the underlying pathogenesis. Clinically, the breakdown or dysfunction of the blood brain barrier can accompany hypoxia, which results in an overall cortical blood flow decrease (20). Group comparison between the MG and the SG showed that significant abnormal blood flow appeared in the bilateral insula and superior medial frontal gyrus.

In the cortical analyses of thickness and blood flow, the insula constantly exhibited abnormality in group comparisons. A previ- ous study showed that the insular cortex is hidden under dense arterial and venous blood vessels (21), making it easily influenced by pneumonia-introduced hypoxia. The deformation and dysfunction of the insula in patients with COVID-19 was obvious. Sufficient evidence has demonstrated that the insula subserves a wide variety of functions in humans, ranging from sensory and affective processing to high-level cognition (21), whereas the precise functional impact of insular abnormality remains unclear. There is also another explanation. For gray matter thickness, we first used 3D-T1 images to segment the whole brain according to gray matter, white matter, and CSF, then used the T1 values to estimate the thickness of the gray matter. If the COVID-19 infection alters the T1 of gray matter, which is possible given the proposed changes in tissue microstructure, then the altered T1 values could make it look like the thickness of the gray matter has changed. Another interesting finding is that in the SG, the CBF in the left insula was positively but not negatively associated with the PCT. The positive correlation possibly implies that the more severe the inflammation is, the more severe the damage is in the insula when patients are hospitalized. As the MRI was conducted 3 months after discharge, we speculated that the more severe the damage was, the faster the recovery would be for severe-type patients. This is a preliminary study and further longitudinal follow-up studies are needed to confirm this speculation. 
Significant tracts volumes between NC and SG
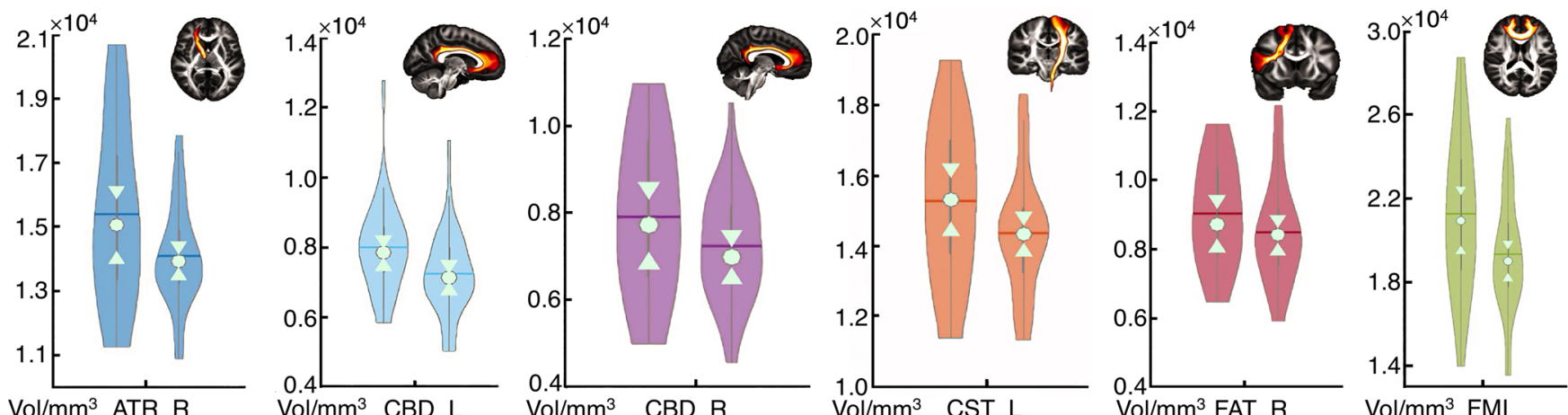

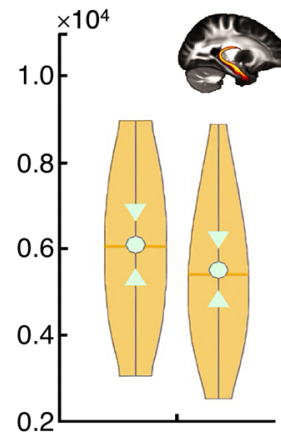

$\mathrm{Vol} / \mathrm{mm}^{3} \quad \mathrm{FX} \mathrm{L}$

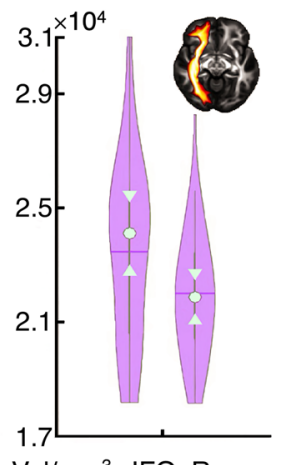

$\mathrm{Vol} / \mathrm{mm}^{3}$ IFO_R

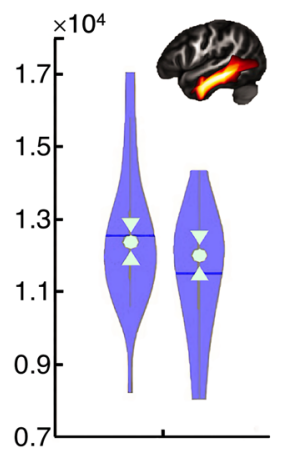

$\mathrm{Vol} / \mathrm{mm}^{3} \quad$ ILFL

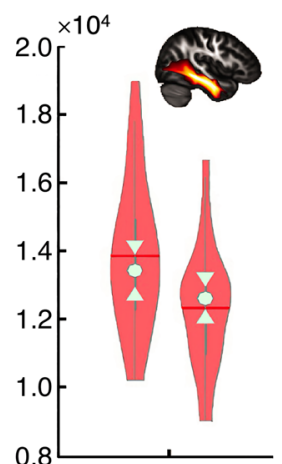

$\mathrm{Vol} / \mathrm{mm}^{3} \quad$ ILF R

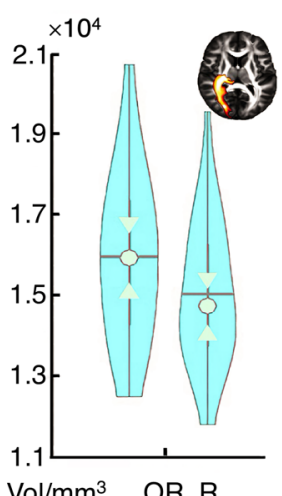

$\mathrm{Vol} / \mathrm{mm}^{3} \quad$ OR_R

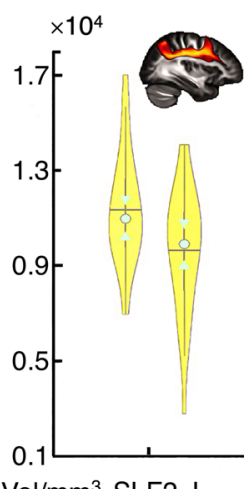

$\mathrm{Vol} / \mathrm{mm}^{3} \mathrm{SLF} 2 \mathrm{~L}$

Figure 5. Significant difference of the tracts' volume between the NC group and the SC. Violin plots for the NC group (left plots, $n=31$ ) and the SG (right plots, $n=32$ ). Permutation test, $P<0.05$. The brain slice of a specific tract from the HCP population is displayed in the right corner. For standardization, these tracts were extracted from the HCP population instead of the subjects' specific tracts.

Subcortical abnormality. Subcortical nuclei analysis of the volume and $\mathrm{CBF}$ also revealed a decreasing pattern that is consistent with cortical thickness analysis, suggesting that the impact of COVID-19 is brain-wide. The bilateral thalamus and left putamen exhibited decreased volume in the SG relative to the NC group. However, there were no subcortical nuclei volume differences between the NC group and the MG, whereas the MG-SG comparison showed abnormality in the left putamen and caudate. The bilateral thalamus is a susceptible target of ANE related to late immune demyelination in COVID-19 (6) and also brain regions sensitive to hypoxemia. The caudate and putamen as parts of the human striatum are distinguished by a marked heterogeneity in functional, anatomical, and neurochemical patterns (22). For CBF comparisons, the SG showed more widespread CBF decrease in the subcortical nuclei mainly located in the striatum and amygdala compared with the MG. These results suggest that COVID-19 severity may elicit different influence, including damage to the critical subcortical nuclei. Follow-up functional analysis will be conducted to address the precise functional abnormality of subcortical nuclei.

For the subcortical white matter tract analysis, we adopted a new robust and reproducible tool, XTRACT, which allows reconstruction of white matter tracts in a consistent manner across subjects, while respecting the underlying anatomical variation and individual differences (23). We found widespread decreases in volume, length, and the mean FA value in associ- ation, commissural, projection, and limbic fiber bundles in the MG and SG when compared with the NC group. In the MG and SG, the volume of several tracts that belong to 4 different fiber bundles decreased when compared with the NC group, with the SG showing more fiber impairment relative to the MG. The significant tracts (right ATR, left CBD, right FAT, FMI, left ILF, right ILF) were overlapped in the NC-MG and NC-SG comparisons. There were no significant differences between the MG and SG, suggesting that COVID-19 impaired the white matter in both the MG and SG to different extents.

For correlational analyses, in the MG, the volume decreases in the right ATR and the right ILF were significantly associated with PCT elevation during hospitalization, indicating that the more severe the disease, the lower the volume of the tracts. This indicates that a high level of inflammatory markers during hospitalization were associated with a high likelihood of volume loss in recovered COVID-19 patients, especially in frontal and limbic systems. This is consistent with the cortical thickness alterations in our study.

There is also another interesting finding in the SG. The volumes of the left CST and right OR and the mean FA value of the left MDLF showed positive correlations with the level of IL-6. Thus, we hypothesized that the white matter could be seriously damaged due to COVID-19 infection, especially for patients with severe disease. The volumes and mean FA value of the white matter tracts could be significantly decreased in several 

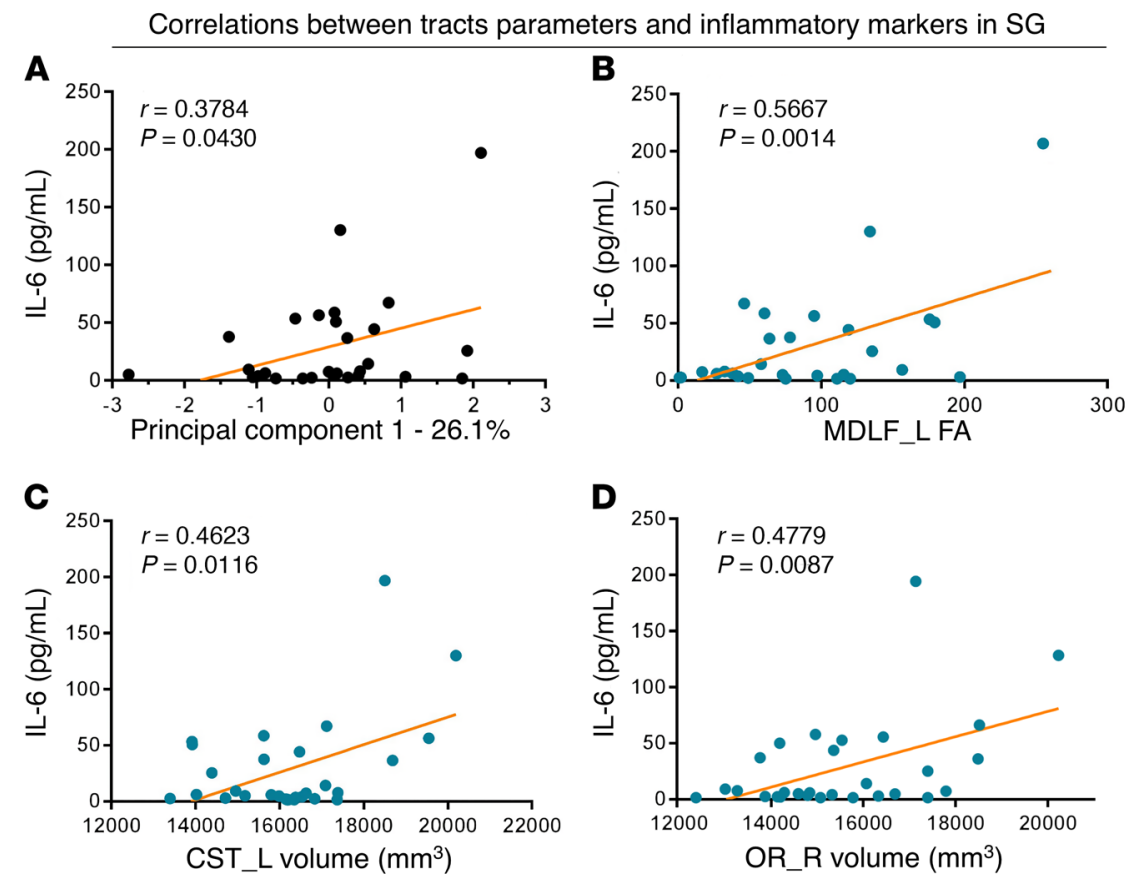

Figure 6. Correlations between the significantly different tracts and inflammatory markers in the SG. Correlations between the PC1 (A), FA of left MDLF (B), volume of the left CST (C), volume of the right $\mathrm{OR}(\mathbf{D})$, and IL-6 respectively. L, left; $\mathrm{R}$, right. $n=32 ; P<0.05$.

brain regions. However, it gradually recovered after patients were cured. The more severe the COVID-19 condition, the more changes in white matter occurred in several brain regions. We speculated that the brain injury in the SG was more severe than that in the MG during hospitalization, as some tracts' recovery rate in the SG was more rapid than in the MG after 3 months of recovery. This is somehow similar to the structural remodeling observed in arrhythmia manifestations of COVID-19 infection (24). Regardless of whether the correlation was positive or negative, the white matter microstructure changes were related to inflammation to some extent. More importantly, in the SG, the connections between brain imaging measurements and inflammatory markers are more obvious than in the MG. However, the underlying mechanisms remain unclear and further quantitative analysis is needed.

Potential mechanism of brain injury. Although totally recovered from pneumonia symptoms, patients who recovered from COVID-19 still exhibited brain microstructure and CBF changes 3 months after recovery. There should be several possible pathogenic mechanisms to explain the influence of COVID-19 on cerebral damage, such as direct viral encephalitis, peripheral organ dysfunction (such as lung, liver, kidney), cerebrovascular changes, and systemic inflammation (25). The dissemination of COVID-19 in the systemic circulation or across the cribriform plate of the ethmoid bone can lead to cerebral involvement, which has been reported in the past for SARS-CoV-affected patients (26). This is the direct mechanism of COVID-19related brain damage during the acute stage reported in previous studies $(2,4,5)$. In our 3-month follow-up study, patients had no specific or acute neurological problems during the acute period of the disease and the major difference between the MG and the SG was that the SG experienced a more severe hypoxic state and inflammatory storm. Correspondingly, the brain imaging measurements in the SG were much more extensive than in the MG, in which just a few changes were found in the white matter tract. The changes in the brain, such as cerebral volumes, cerebral blood flow, and white matter tracts, were highly correlated with inflammatory factors in the SG. Thus, we assumed that the main underlying mechanism could be related to indirect injury due to an inflammatory storm induced by the immune response (27) or hypoxia. Furthermore, neuropathologic features of autopsied COVID-19 showed various hypoxia injury in the brain, such as cell-abundant enlarged perivascular spaces, with no signs of encephalitis or meningitis $(28,29)$. Neurochemical measurements showed that patients with COVID-19 with different severity states had varying degrees of neuronal injury and glial activation, supporting that indirect injury is the main mechanism and not the direct invasion of the virus (13). On the other hand, hypercoagulability and microembolization of the vascular endothelium may also be implicated, as evidenced by the hypoperfusion on arterial spin label (ASL) across the gray matter cortex in patients with severe-type disease. In our limited cases, a small number of patients with severe-type disease also had kidney or heart damage, leading us to believe that the multi-organ injury could be another possible mechanism. Taken together, all those observations point to the indirect injury mechanism in the brain during long-term recovery.

Limitations. There are several limitations in the present study. First, the sample size is small. We should aim to increase the sample size in a further follow-up study. Second, patients had no specific neurological manifestation and so, to avoid cross-infection, the head MRI was not performed during the acute phase. Third, a follow-up study should be conducted to see whether brain anatomical and functional changes progress or regress.

Conclusion. In this study, gray matter atrophy, widespread CBF reduction, and white matter microstructure changes detected by the quantitative MRI technique were found in patients recovered from COVID-19 pneumonia, providing new evidence to the neurological damage of COVID-19 on long-term recovery. The abnormalities in these brain areas should be monitored in the process of complete recovery, which would help clinicians understand the potential neurological sequelae of COVID-19.

\section{Methods}

Participants. Patients who recovered from COVID-19 pneumonia were recruited from the Department of Infectious Disease in Tongji Hospital, with the inclusion criteria as follows: (a) 50-70 years old; (b) diagnosed as having mild- or severe-type COVID-19 according to WHO interim guidance during hospitalization and did not require a stay in intensive care unit; (c) no specific neurolog- 


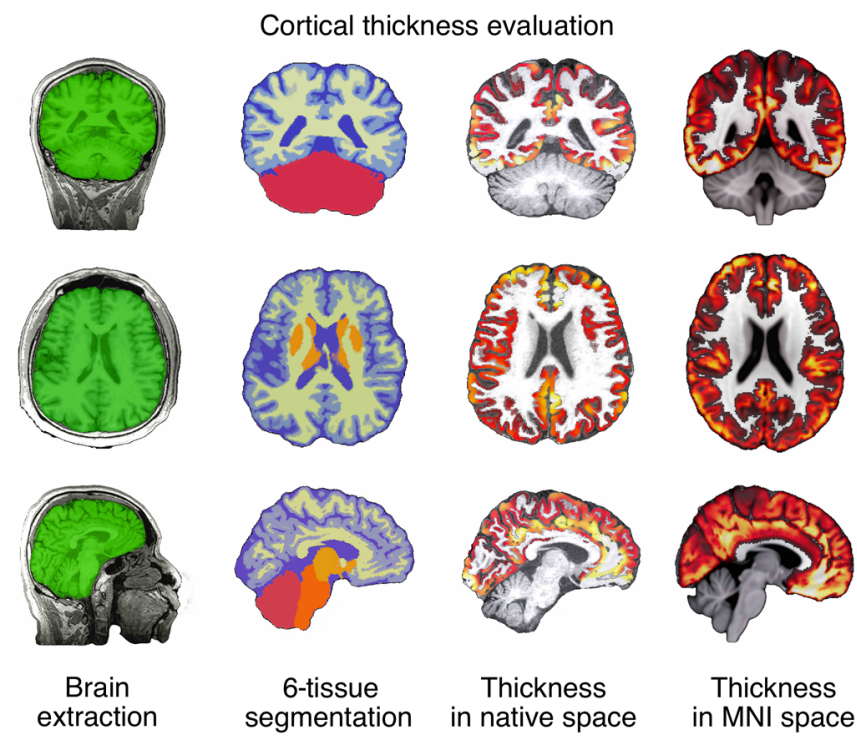

Figure 7. Workflow of ANTs cortical thickness estimation. ANTs cortical thickness pipeline yielded clear-cut brain extraction and 6-tissue segmentation, as well as nearly perfect estimation of thickness in the native space and accurate warping into the MNI space.

ical manifestations during the acute stage, such as smell/vision/ hearing/taste/memory loss, seizures, and impaired mobility; (d) no specific neurological manifestations and no obvious lesion on the conventional MRI for 3 months after hospital discharge; (e) no history of head trauma, stroke, brain tumors, metabolic disturbance, or epilepsy; and (f) blood sample was available to get the hematocrit value before the MRI scan. The clinical information of patients with COVID-19 was obtained from their hospital records and included clinical type and inflammatory markers such as CRP, PCT, and IL-6. Age-, sex-, and education-matched healthy volunteers were recruited from the local community, excluding those with previous history of head trauma, stroke, brain tumors, metabolic disturbance, and epilepsy. Also, polycythemia and anemia were excluded to ensure the accuracy of CBF calculation. All participants were fully informed about the purpose of the study and agreed to take the head MRI and chest CT scan and completed questionnaires that included age, sex, education years, handedness, and underlying diseases on the same day.

Blood test. The levels of PCT and IL- 6 were detected using an automatic Biochemical analyzer (Roche Cobas e602). The levels of CRP (IATRON) were detected using a biochemical analyzer (Roche Cobas 8000). The hematocrit tests (routine blood test) were performed using the Sysmex Automated Hematology Analyzer (XS 500i).

MRI scan. The MRI scan was performed using a 3.0 T MR scanner (Discovery MR750, GE Healthcare) with a 32-channel head array coil. The protocol included conventional MRI, structural 3D T1-weighted images (3D-T1WIs), high-resolution diffusion tensor imaging (DTI), and 3D pseudo-continuous arterial spin labeling (3D-pcASL). Foam padding and ear plugs were used to reduce head motion and scanner noise.

The conventional MRI protocol included axial T2-FLAIR sequence with TR/TE/TI of $8400 / 165 / 2100 \mathrm{~ms}$, matrix size of 256 $\times 256$, FOV of $240 \times 240 \mathrm{~mm}^{2}$, slice thickness/gap of $5 / 1.5 \mathrm{~mm}$, and number of slices equal to 16 .
3D-T1WIs were acquired using brain volume (BRAVO) sequence with TR/TE/TI of 7.1/2.7/450 ms, flip angle of $12^{\circ} \mathrm{C}$, matrix size of 256 $\times 256$, FOV of $240 \times 280 \mathrm{~mm}^{2}$, number of averages equal to 1 , slice thickness of $1 \mathrm{~mm}$, and number of slices equal to 184 .

DTI was obtained using a single-shot diffusion-weighted echo-planar imaging (EPI) sequence in the axial plane with TR/TE of $8500 / 60.4 \mathrm{~ms}$, matrix size of $128 \times 128$, FOV of $256 \times 256 \mathrm{~mm}$, slice thickness/gap of $2 / 0 \mathrm{~mm}, b$ value of $1000 \mathrm{~s} / \mathrm{mm}^{2}$ along 64 directions, $10 \mathrm{~b}_{0}$ images, and number of slices equal to 70 .

3D-pcASL was acquired using an interleaved 3D stack of spirals fast spin echo (FSE) sequence with a high-level background suppression to cover the whole brain. The detailed parameters were as follows: TR/TE of 4788/14.6 ms, postlabeling delay (PLD) of $1525 \mathrm{~ms}$, number of averages equal to 3 , bandwidth of $62.5 \mathrm{kHz}$, readout of $8 \mathrm{arms} \times$ 1024 samples, FOV of $240 \times 240 \mathrm{~mm}$, slice thickness/gap of $4 / 0 \mathrm{~mm}$, and number of slices equal to 34 . The labeling duration was $1500 \mathrm{msec}$ and the resolution was $4 \times 4 \times 4 \mathrm{~mm}$.

To ensure the robustness of our analyses, image quality control was strictly performed from acquisition to preprocessing. The quality of the signal fidelity, brain extraction, and registration in each stage on every image were plotted and visually checked. Subjects with signal loss, artefacts, poor registration, and large head motion were excluded from further analysis. In the current study, no subjects were excluded due to poor quality image and registration. Two healthy volunteers were excluded due to signal loss in the cerebellum of raw images.

MRI data preprocessing: cortical thickness evaluation. To obtain accurate native space skull-stripped brain masks, segmentations, and MNI space-warped subcortical nuclei volumes, 3D-T1WIs were uploaded to the online anatomical segmentation pipeline volBrain (30). According to our prior experience and visual check, the machine-learning-based volBrain pipeline produced clear-cut gray matter, white matter, cerebrospinal fluid (CSF), and subcortical nuclei segmentations, and the results were much better than other algorithms such as BET in FSL (FMRIB Software Library, ref. 31), FreeSurfer (32), and ANTs (Advanced Normalization Tools, http:// stnava.github.io/ANTs/). The native brain masks and subcortical segmentations were used in the following analyses.

The cortical thickness evaluation was conducted using ANTs (33). The automated, volume-based ANTs cortical thickness pipeline was comprised of well-vetted components such as SyN (image registration), N4 (bias correction), Atropos (n-tissue segmentation), and DiReCT (cortical thickness estimation). The ANTs cortical thickness estimation workflow is illustrated in Figure 7. The steps were as follows: (a) initial N4 bias correction on T1 images, (b) brain extraction using a segmentation/template-based strategy (i.e., 6 segments priors: cerebrospinal fluid prior, gray matter prior, white matter prior, subcortical nuclei prior, cerebellum prior, brainstem prior) as specified before (34), (c) alternation between prior-based segmentation and pure tissue posterior probability weighted bias correction using Atropos and N4, (d) DiReCT-based cortical thickness estimation, and (e) normalize to MNI $1 \mathrm{~mm}$ resolution template.

pcASL preprocessing. All raw pcASL images were transferred to the workstation (Advantage Workstation 4.6, GE Medical Systems) to obtain the native CBF map according to the kinetic model proposed by Alsop and Detre (35). In addition, we included a term for 
Seed Target $\square$ Exclude Stop

A

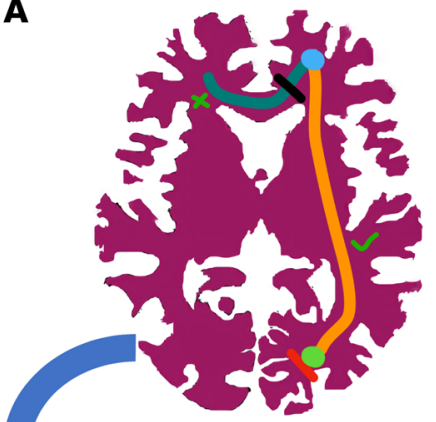

Warp protocols to native space and run tractography

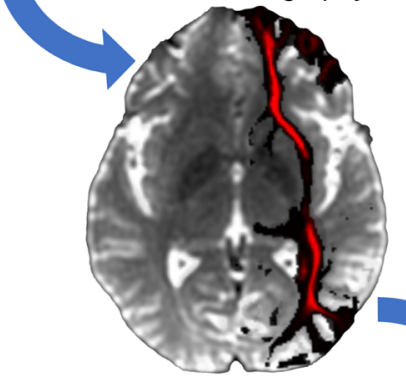

Warp results into standard space

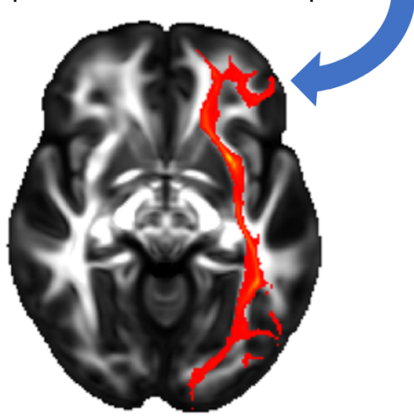

B
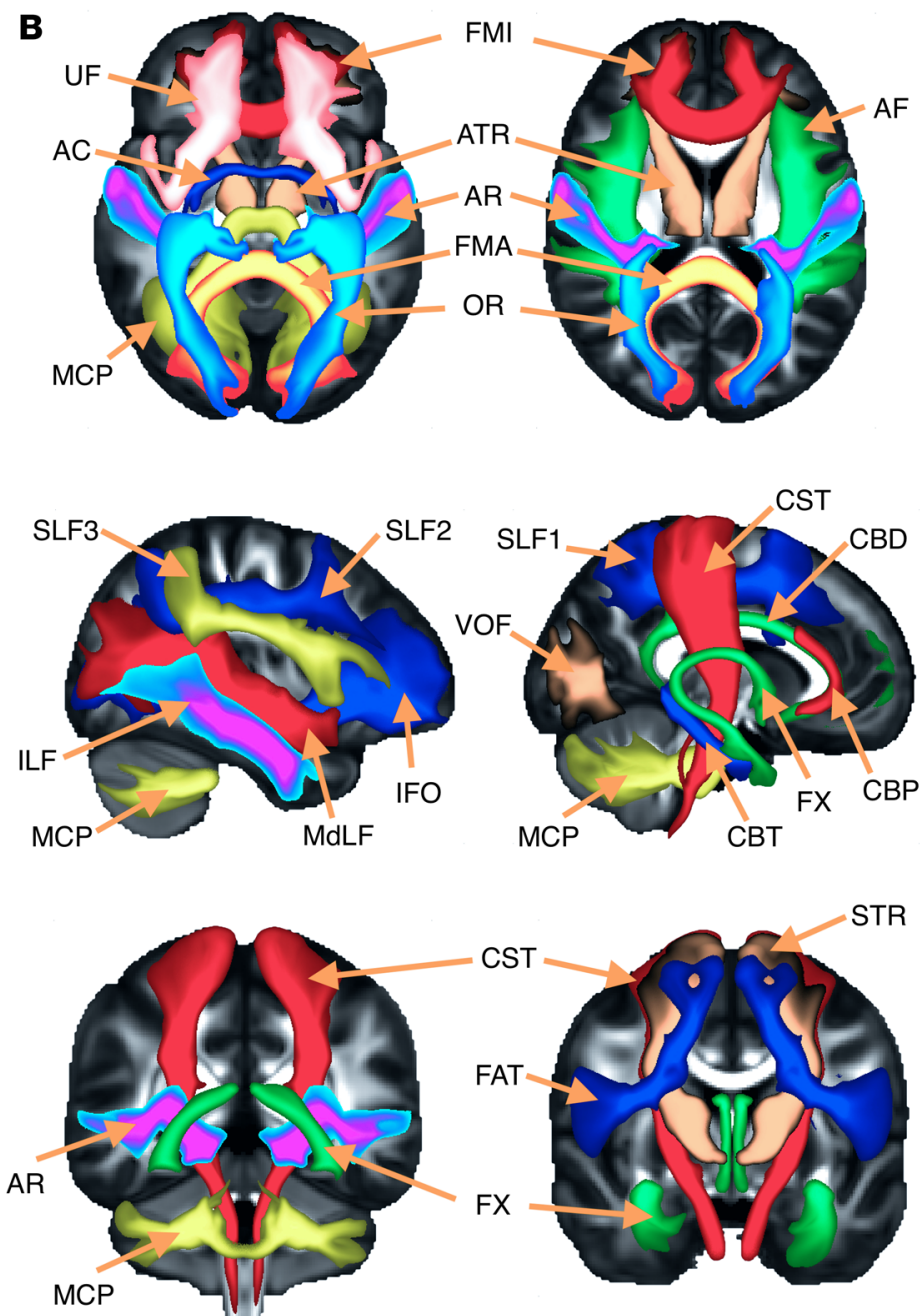

Figure 8. Illustration of the method of XTRACT automated tractography. (A) Diagram of the steps for the XTRACT automated tractography, with an example of the left IFO. Tractography protocol masks were defined in standard space (FSL_HCP1065 FA atlas, derived from the HCP data set) with seed (start point, blue), exclusion (rejection, black), target (destination, green), and stop (termination, red) masks. The native and standard tractography maps were extracted from a subject from the current sample. (B) Illustration of the projections of the HCP population tract atlases. Association fiber bundles: arcuate fascic-ulus (AF), FAT, ILF, IFO, middle longitudinal fasciculus (MdLF), SLF1, 2, and 3, uncinate fasciculus (UF), and vertical occipital fasciculus (VOF). Projection fiber bundles: AR, ATR, CST, OR, and superior thalamic radiation (STR). Limbic fiber bundles: cingulum bundle, perigenual (CBP), cingulum bundle, temporal (CBT), CBD, and FX. Commissural fiber bundles: anterior commissure (AC), forceps major (FMA), and FMI.

the finite labeling duration and corrected the incomplete recovery of the tissue signal in the reference image due to the saturation performed $t_{\text {sat }}$ (2000 msec) before imaging (36). The quantitative CBF maps were based on the following equation:

$$
C B F=\frac{\lambda}{2 \alpha \mathrm{T}_{1 \beta}\left(1-\mathrm{e}^{\frac{\tau}{T_{1 \beta}}}\right)} \frac{\left(S_{c t r l}-S_{l b l}\right)\left(1-\mathrm{e}^{\frac{\tau_{s t g}}{T_{t g}}}\right)}{\mathrm{e}^{\frac{\omega}{T_{1 \beta}}}}
$$

Equation 1

where $S_{c t r r}, S_{l b l}, S_{r e f}$ are the signal on the control, label, and reference image; $T_{1 b}$ is the T1 relaxation time of the blood at 3.0T about 1600 msec; $T_{1 g}$ represents the $\mathrm{T} 1$ relaxation time of the gray matter at 3.0T about $1200 \mathrm{msec} ; t_{\text {sat }}$ is the time of saturation performed before imaging about $2000 \mathrm{msec} ; \alpha$ is the labeling efficiency of 0.8 ; $\lambda$ is cortex-blood partition coefficient of 0.9 ; $\tau$ is the labeling duration about $1500 \mathrm{msec}$; and $\omega$ is the PLD time of $1525 \mathrm{msec}$.

The preprocessed native $\mathrm{CBF}$ images were aligned to the $\mathrm{T} 1$ images using FLIRT pipeline in FSL tools and then warped into the MNI space using subject-specific ANTs warp fields previously produced by $\mathrm{T} 1$ to MNI template nonlinear registration. To obtain the subcortical nuclei CBF, the subcortical nuclei masks in the MNI space were obtained from volBrain, and the masks were used to extract the subcortical nuclei CBF.

DTI preprocessing. The DTI images were preprocessed using FDT pipeline in the FSL. The steps were as follows: (a) first, correcting of the diffusion data for eddy current-induced distortions and subject movements (EDDY), (b) the first BO image was brain- 
extracted (BET) to remove the extra tissues, and then the generated mask was applied to the rest of the diffusion data; (c) fitting of the diffusion tensors on corrected data (DTIFIT); (d) fitting of the probabilistic diffusion model on corrected data (Bayesian Estimation of Diffusion Parameters Obtained using Sampling Techniques [BEDPOSTX]); (e) using the first BO image as the reference, linearly registered the $\mathrm{BO}$ image to $\mathrm{T} 1$ image (FLIRT); (f) spatial normalization from $\mathrm{T} 1$ to the MNI space (FNIRT); and (g) finally, performing a newly developed pipeline to automatically extract a set of carefully dissected tracts (Figure 8A) in the subject's native space using probabilistic diffusion tractography (23).

XTRACT is a new software package with a library of standardized tractography protocols (BEDPOSTX and PROBTRACKX) devised for the robust automated extraction of white matter (Figure 8B). Deriving data from the Human Connectome Project (HCP) and the UK Biobank, white matter atlases and atlases for tract endpoints on the white-gray matter boundary were obtained. These automated ROI-based tractography approaches have proven powerful in the extraction of a range of tracts. The tractography reflects the known anatomy and prior anatomical knowledge used to guide and constrain curve propagation, reducing the chance of false positives $(37,38)$.

In the algorithm BEDPOSTX, the $\mathrm{X}$ stands for modeling crossing fibers within each voxel of the brain. BEDPOSTX runs Markov Chain Monte Carlo sampling to build up distributions on diffusion parameters at each voxel. Crucially, BEDPOSTX automatically determines the number of crossing fibers per voxel. It creates all the files necessary for running probabilistic tractography PROBTRACKX.

PROBTRACKX produces sample tractographies, by starting from some seeds and then iterate between drawing an orientation from the voxel-wise BEDPOSTX distributions, taking a step in this direction, and checking for any termination criteria. These sample tractographies can then be used to build up a histogram of how many tractographies visited each voxel or the number of tractographies connecting specific brain regions. The probabilistic estimation of multifiber tractography at each voxel offers significant advantages in sensitivity when tracking nondominant fiber populations but does not dramatically change tractography results for the dominant pathways.

Statistics. To ensure statistical consistency, all comparisons were conducted using the permutation test (5000 random shuffles of subject labels, $P<0.05)$. The statistical analyses on the cortical thickness images and pcASL CBF images were performed using the permutation test (randomize in FSL) (39) with the threshold-free cluster enhancement (TFCE).

The statistical comparison of subcortical nuclei volumes, subcortical CBF values, and tract statistics was performed using custom code in MATLAB (R2020a, The MathWorks, Inc.). Specifically, for a given nucleus/tract in one group comparison, the difference of the given nucleus/tract between each group was calculated (i.e., the real statistic and then 5000 random shuffles of subject labels were performed). We then recalculated the difference in each shuffle iteration to generate a permutation distribution, and finally the upper threshold is the real statistic at 95 percent of the permutation distribution (40). Five thousand permutations were demonstrated to be robust for generating the null distribution, which yielded no differences when set to 10000 permutations. Due to consideration of the age effect on subcortical nuclei, age was regressed out before the permutation test. However, we found that whether regressed out or not, age had no influence on the results in the permutation test.

To further explore the relationships between the MRI image features and inflammatory biomarkers, we first regressed out the age effect on the resultant significant $(P<0.05)$ cortical brain regions, subcortical nuclei volumes, subcortical $\mathrm{CBF}$ values, and tract volume/length/FA. Then, the principal component analysis (PCA) was used to reduce the dimensionality of the significant different imaging results. As a common statistical multivariate approach, PCA can generate a new set of variables (principal components [PCs]) with minimal loss of information (41). The PCs are generated with a linear combination of the original variables and generate a low-dimensional space which preserves most of the data variance. The first component (PC1) was correlated (Pearson correlation) with the inflammatory marker values. To explore the contribution of the significant changes in imaging results, the imaging results were further correlated (Pearson correlation) with the values of the upper significant findings related to inflammatory markers. $P$ less than 0.05 was considered statistically significant in both cases. Categorical data were compared using the Fisher exact test. Demographic and behavior measures were compared using the Student's 2-tailed $t$ test.

Study approval. This cross-sectional study was approved by the Clinical Institute Ethics Committee of Tongji Hospital (Wuhan, China). Written informed consent was obtained from each participant.

\section{Author contributions}

WZ and J Wang had full access to all of the data in the study and take responsibility for the integrity of the data and the accuracy of the data analysis. YQ, J Wu, J Wang, WZ conceived and designed the study. J Wu, YQ, JL, TC, J Wang, and WZ acquired, analyzed, and interpreted data. YQ, J Wu, J Wang, and WZ drafted the manuscript. AM, FX, and QN critically revised the manuscript for important intellectual content. $\mathrm{J} \mathrm{Wu}$ and JL performed statistical analysis. WZ obtained funding. GZ, DW, YZ, NZ, and AC contributed administrative, technical, and material support. WZ and J Wang supervised the study. The order of the co-first authors was determined by the extent of their contributions to the work.

\section{Acknowledgments}

We would like to express our gratitude to the statistician Chaolong Wang (School of Public Health, Huazhong University of Science and Technology) for his valuable contributions during statistical methods discussions. This study was funded by the key projects of the Natural Science Foundation of China (81730049).

Address correspondence to: Jie Wang, Innovation Academy for Precision Measurement Science and Technology, Chinese Academy of Sciences, Wuhan, Hubei 430071, China. Phone: 86.27.87197653; Email: jie.wang@apm.ac.cn. Or to: Wenzhen Zhu, Department of Radiology, Tongji Hospital, Tongji Medical College, Huazhong University of Science and Technology, 1095 Jiefang Avenue, Wuhan, 430030, China. Phone: 86.27.83663258; Email: zhuwenzhen8612@163.com. 
1. WHO. Weekly Epidemiological Update - 29 December 2020. http://who.int/publications/m/ item/weekly-epidemiological-update---29-december-2020. December 29, 2020. Accessed April 2, 2021.

2. Mao L, et al. Neurologic manifestations of hospitalized patients with coronavirus disease 2019 in Wuhan, China. JAMA Neurol. 2020;77(6):683-690.

3. Pezzini A, Padovani A. Lifting the mask on neurological manifestations of COVID-19. Nat Rev Neurol. 2020;16(11):636-644.

4. Moriguchi T, et al. A first case of meningitis/ encephalitis associated with SARS-coronavirus-2. Int J Infect Dis. 2020;94:55-58.

5. Huang YH, et al. SARS-CoV-2 detected in cerebrospinal fluid by PCR in a case of COVID-19 encephalitis. Brain Behav Immun. 2020;87:149.

6. Poyiadji N, et al. COVID-19-associated acute hemorrhagic necrotizing encephalopathy: imaging features. Radiology. 2020;296(2):E119-E120.

7. Parsons T, et al. COVID-19-associated acute disseminated encephalomyelitis (ADEM). J Neurol. 2020;267(10):2799-2802.

8. Hernandez-Fernandez F, et al. Cerebrovascular disease in patients with COVID-19: neuroimaging, histological and clinical description. Brain. 2020;143(10):3089-3103.

9. Zhao H, et al. Guillain-Barre syndrome associated with SARS-CoV-2 infection: causality or coincidence? Lancet Neurol. 2020;19(5):383-384.

10. Gutierrez-Ortiz C, et al. Miller Fisher syndrome and polyneuritis cranialis in COVID-19. Neuro$\operatorname{logy}$. 2020;95:e601-e605.

11. Koralnik IJ, Tyler KL. COVID-19: a global threat to the nervous system. Ann Neurol. 2020;88(1):1-11.

12. Enkirch SJ, et al. The ERICA score: an MR imaging-based visual scoring system for the assessment of entorhinal cortex atrophy in Alzheimer disease. Radiology. 2018;288(1):226-333.

13. Kanberg N, et al. Neurochemical evidence of astrocytic and neuronal injury commonly found in COVID-19. Neurology. 2020;95(12):e1754-e1759. 14. Xiong W, et al. New onset neurologic events in people with COVID-19 in 3 regions in China. Neurology. 2020;95(11):e1479-e1487.

15. Lindlau A, et al. Predictors of hippocampal atrophy in critically ill patients. Eur J Neurol. 2015;22(2):410-415.

16. Bellmund JLS, et al. Navigating cognition: spatial codes for human thinking. Science. 2018;362(6415):eaat6766.

17. Kim EJ, et al. Stress effects on the hippocampus: a critical review. Learn Mem. 2015;22(9):411-416.

18. Kremer S, et al. Brain MRI findings in severe COVID-19: a retrospective observational study. Radiology. 2020;297(2):E242-E251.

19. Helms J, et al. Neurologic features in severe SARS-CoV-2 Infection. $N$ Engl JMed. 2020;382(23):2268-2270.

20. Kandemirli SG, et al. Brain MRI findings in patients in the intensive care unit with COVID-19 infection. Radiology. 2020;297(1):E232-E235.

21. Uddin LQ, et al. Structure and function of the human insula. JClin Neurophysiol. 2017;34(4):300-306.

22. Hortnagl $\mathrm{H}$, et al. Distinct gradients of various neurotransmitter markers in caudate nucleus and putamen of the human brain. J Neurochem. 2020;152(6):650-662.

23. Warrington S, et al. XTRACT - standardised protocols for automated tractography in the human and macaque brain. Neuroimage. 2020;217:116923.

24. Babapoor-Farrokhran S, et al. Arrhythmia in COVID-19. SN Compr Clin Med. 2020;2:1430-1435.

25. Heneka MT, et al. Immediate and long-term consequences of COVID-19 infections for the development of neurological disease. Alzheimers Res Ther. 2020;12(1):69.

26. Baig AM, et al. Evidence of the COVID-19 virus targeting the CNS: tissue distribution, host-virus interaction, and proposed neurotropic mechanisms. ACS Chem Neurosci. 2020;11(7):995-998.

27. Huang C, et al. Clinical features of patients infected with 2019 novel coronavirus in Wuhan, China. Lancet. 2020;395(10223):497-506.
28. Solomon IH, et al. Neuropathological features of Covid-19. N Engl J Med. 2020;383(10):989-992.

29. Kantonen J, et al. Neuropathologic features of four autopsied COVID-19 patients. Brain Pathol. 2020;30(6):1012-1016.

30. Manjon JV, Coupe P. volBrain: an online MRI brain volumetry system. Front Neuroinform. 2016;10:30.

31. Jenkinson M, et al. FSL. Neuroimage. 2012;62(2):782-790.

32. Fischl B. FreeSurfer. Neuroimage. 2012;62(2):774781.

33. Tustison NJ, et al. Large-scale evaluation of ANTs and FreeSurfer cortical thickness measurements. Neuroimage. 2014;99:166-179.

34. Avants BB, et al. An open source multivariate framework for $n$-tissue segmentation with evaluation on public data. Neuroinformatics. 2011;9(4):381-400.

35. Alsop DC, Detre JA. Reduced transit-time sensitivity in noninvasive magnetic resonance imaging of human cerebral blood flow. J Cereb Blood Flow Metab. 1996;16(6):1236-1249.

36. Wu B, et al. Intra- and interscanner reliability and reproducibility of 3D whole-brain pseudo-continuous arterial spin-labeling MR perfusion at 3T. JMagn Reson Imaging. 2014;39(2):402-409.

37. Catani M, et al. Virtual in vivo interactive dissection of white matter fasciculi in the human brain. Neuroimage. 2002;17(1):77-94.

38. Wakana S, et al. Fiber tract-based atlas of human white matter anatomy. Radiology. 2004;230(1):77-87.

39. Winkler AM, et al. Permutation inference for the general linear model. Neuroimage. 2014;92:381-397.

40. Nichols TE, Holmes AP. Nonparametric permutation tests for functional neuroimaging: a primer with examples. Hum Brain Mapp. 2002;15(1):1-25.

41. Higgins V, et al. Principal component and correlation analysis of biochemical and endocrine markers in a healthy pediatric population (CALIPER). Clin Biochem. 2019;66:29-36. 